\title{
A propensity-matched comparison of cost and outcomes after esophageal stent placement or primary surgical repair for iatrogenic esophageal perforation
}

\author{
Richard K. Freeman, MD, MBA, ${ }^{a}$ Argenis Herrera, MD, ${ }^{b}$ Anthony J. Ascioti, MD, ${ }^{a}$ \\ Megan Dake, PA-C, ${ }^{\text {a }}$ and Raja S. Mahidhara, MD
}

\begin{abstract}
Objectives: Esophageal stent placement has been shown to be a safe and effective treatment for acute esophageal perforation in selected patients. However, a comparison between surgical repair and stent placement has not been reported. This investigation compares the outcomes and costs of the 2 treatment modalities.
\end{abstract}

\begin{abstract}
Methods: The Premiere database for a single health system's hospitals was used to identify patients undergoing treatment for an acute intrathoracic esophageal perforation over a 4-year period. Patient cohorts for stent placement or surgical repair were formed using propensity matching. The 2 cohorts were compared for length of stay, morbidity, mortality, and costs.
\end{abstract}

Results: Between 2009 and 2012, 60 patients undergoing esophageal stent placement or surgical repair were propensity matched. Mean patient age and Charlson comorbidity scores did not differ significantly $(P=.4$ and $P=.4$, respectively). Significant differences in morbidity $(4 \%$ vs $43 \% ; P=.02)$, mean length of stay (6 vs 11 days; $P=.0007)$, time to oral intake (3 vs 8 days; $P=.0004)$, and cost $(\$ 91,000$ vs $\$ 142,000 ; P<.0001)$ were identified in the esophageal stent cohort when compared with patients receiving surgical repair. Operative mortality did not differ significantly.

Conclusions: Esophageal stent placement for the treatment of an acute esophageal perforation seems to be as effective as surgical repair when compared between propensity-matched patients. However, stent placement resulted in a shorter length of stay, lower rates of morbidity, and lower costs when compared with traditional surgical repair. (J Thorac Cardiovasc Surg 2015;149:1550-5)

See related commentary pages $1556-7$.

Over the last decade, esophageal stent placement for the treatment of esophageal perforation, fistula, and anastomotic leak has been found to be beneficial in selected patients. Specifically, a hybrid approach to patients with esophageal perforation using esophageal stent placement, enteral nutrition, and minimally invasive surgery, when required, has been described by several groups. ${ }^{1-3}$ However, because of the relative rarity of esophageal perforation, the significant diversity of the patient population and the concern by some investigators that

From the Department of Thoracic and Cardiovascular Surgery ${ }^{\mathrm{a}}$ and General Surgery, ${ }^{\mathrm{b}}$ St Vincent Hospital, Indianapolis, Ind.

St Vincent Health provided the sole source of funding for this investigation.

Read at the 94th Annual Meeting of The American Association for Thoracic Surgery, Toronto, Ontario, Canada, April 26-30, 2014.

Received for publication April 10, 2014; revisions received Jan 11, 2015; accepted for publication Jan 23, 2015; available ahead of print March 17, 2015.

Address for reprints: Richard K. Freeman, MD, MBA, 8433 Harcourt Rd, Indianapolis, IN 46260 (E-mail: Richard.Freeman@StVincent.org).

$0022-5223 / \$ 36.00$

Copyright (c) 2015 by The American Association for Thoracic Surgery

http://dx.doi.org/10.1016/j.jtcvs.2015.01.066 equipoise no longer exists between the 2 treatment strategies, a randomized comparison of patients treated with surgical repair or esophageal stent placement has not been published. The objective of this study was to compare the patient outcomes and costs of treatment between propensity-matched patients undergoing transthoracic operative repair (OR) or esophageal stent repair (SR) for the treatment of an intrathoracic iatrogenic esophageal perforation.

\section{MATERIAL AND METHODS}

The study institution's institutional review board approved this protocol, including the "off-label" use of esophageal stenting, and waived individual patient consent for this investigation with the condition of patient anonymity outside the initial data gathering phase of the study. Patients undergoing surgical repair or esophageal stent placement (Current Procedural Terminology code 43415, 43256) for an iatrogenic, intrathoracic esophageal perforation (diagnosis codes 530.4, 862.22; International Classification of Diseases, Ninth Revision) during the calendar years 2009 to 2012 within the Ascension Health system by a cardiothoracic surgeon were identified using the Premier inpatient database (Premier Inc, Charlotte, NC) (Figure 2). Although the study population was derived only from Ascension Health facilities so that cost data could be analyzed, the Premier Perspective database covers $20 \%$ of the US hospital discharges. Among other things, it is the largest inpatient drug use database in the United States. The database contains complete billing and coding history on more than 45 million hospital inpatient discharges and more than 210 million hospital outpatient visits "from acute care facilities, ambulatory surgery centers and clinics across the nation." Also calculated 


\section{Abbreviations and Acronyms \\ $\mathrm{OR}=$ operative repair \\ $\mathrm{SR}=$ stent repair}

were Charlson Comorbidity scores for each patient, based on secondary International Classification of Diseases, Ninth Revision disease codes. ${ }^{4,5}$

Excluded from further analysis were patients with a cervical or intra-abdominal esophageal perforation, patients with an esophageal malignancy, and patients with an esophageal perforation resulting from other causes. Also excluded were patients undergoing procedures other than primary surgical repair with or without buttressing of the repair or those treated with a self-expanding metallic stent. This included patients undergoing esophageal diversion or exclusion and patients undergoing esophagectomy or reoperative repair as an initial procedure. Eligibility also included the availability of 6-month follow-up for a patient after the treatment of their esophageal perforation.

The propensity score method was used to populate 2 patient cohorts from the patients identified: those undergoing OR and those undergoing SR. ${ }^{6,7}$ Propensity scores were computed after multivariable regression analysis assessing a set of preoperative risk factors that included year of treatment, location of treatment, age, gender, Charlson comorbidity score, and time from perforation to treatment. Patients who underwent SR were matched nearest neighbor in a 1:1 fashion to those who had OR on the basis of the propensity score so that only patients with similar scores were compared.

Patient demographic data, intensive care unit and total length of hospital stay, readmission, operative morbidities, and mortality were abstracted for each patient from the Premier database. Postoperative morbidities were attributed in a binary fashion by patient so that a patient having more than 1 event was counted only once. However, the tabulation of specific categories of complications includes every occurrence.

Sepsis was defined by the presence of a fever, leukocytosis, arterial hypotension, and organ dysfunction. Operative mortality was defined as patient death after surgery before discharge from the hospital or within 30 days of surgery. All health care provided after discharge from the index admission related to the esophageal perforation for a period of 6 months was reviewed. A readmission was defined as any unplanned admission to any hospital within 30 days of the date of discharge from the initial admission. Dysphagia after esophageal perforation repair was defined as significant if it required endoscopic treatment.

\section{Costs}

Costs for individual patients were obtained from the Premier database. Inpatient and outpatients costs attributable to the esophageal perforation are reported. This included facility costs after discharge for inpatient and outpatient care, as well as any outpatient procedures, imaging, or readmissions to the hospital related to the esophageal perforation. For patients undergoing SR, the costs attributed to stent removal were included in the costs of their treatment. Not included in total costs are any approximations of provider costs.

Inpatient costs are broken down into traditional categories, including surgery, room and board, supplies and equipment, intensive care unit, respiratory therapy, laboratory, pharmacy, imaging, physiotherapy/ occupational therapy/speech, and other. Costs were reported as averages, and all categories include direct and indirect costs to the hospital because indirect costs could not be separated retrospectively by facility. Cost parity was assumed because each hospital was in the same health care system with a common supply chain and vendor list.

Bivariate analysis of data was performed using GraphPad Prism software 4.02 (GraphPad Software Inc, San Diego, Calif) for Windows

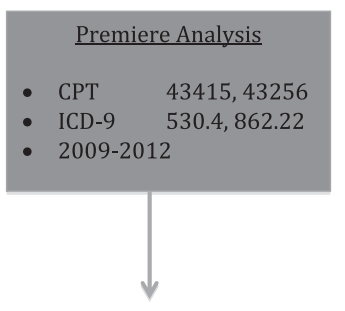

$\begin{array}{ll}\text { Perforation } & \text { Surgical Repair } \\ 57 & 163\end{array}$
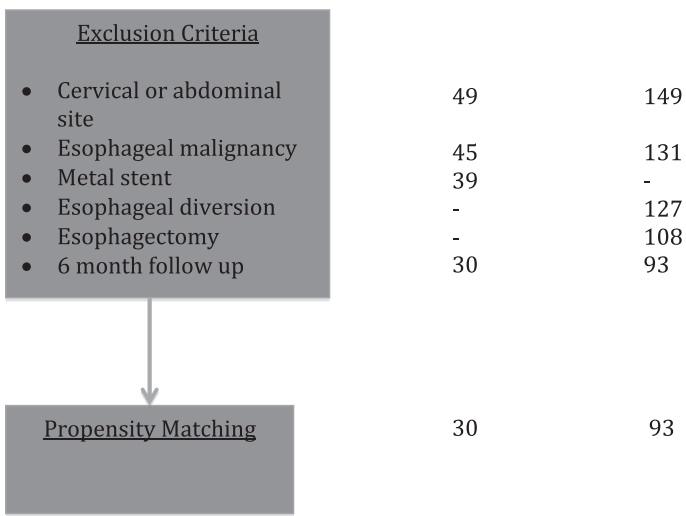

FIGURE 1. Consort table of propensity matching. $C P T$, Current Procedural Terminology; ICD-9, International Classification of Diseases, Ninth Revision.

(Microsoft Corp, Redmond, Wash). Differences between categoric variables were evaluated by the Fisher exact test. Differences between continuous variables were measured by the 2-tailed Student $t$ test or the Mann-Whitney test for non-normally distributed data. Multivariate analysis and propensity matching were performed using Stata version 11 (StataCorp, LP, College Station, Tex).

\section{RESULTS}

During the 4-year study period, 30 patients were identified who met the previous outlined criteria for SR and were propensity matched to 30 of the 93 patients who underwent OR during the same time period. Patients undergoing SR came from 3 institutions, and patients undergoing OR were matched from 6 different facilities. Figure 1 displays the propensity matching process used to form the SR and OR treatment cohorts. Table 1 compares their demographic data, which implies they are comparable on the basis of the results of the matching process. The cause of perforation is also listed. Comparable number of patients from each cohort who met the clinical criteria for a diagnosis of sepsis on admissions.

Table 2 shows the summary measures of the OR and SR treatment strategies, such as muscle buttressing in the OR group or associated procedures in the SR group. The majority of patients in each treatment group had enteral feeding access established. This was with jejunostomy most commonly in the OR group and percutaneous endoscopic gastrostomy in the SR group. Nineteen patients in the SR group underwent a video-assisted thoracoscopic procedure to drain the mediastinum or pleural space at the time of stent placement, whereas 3 patients in the OR group 

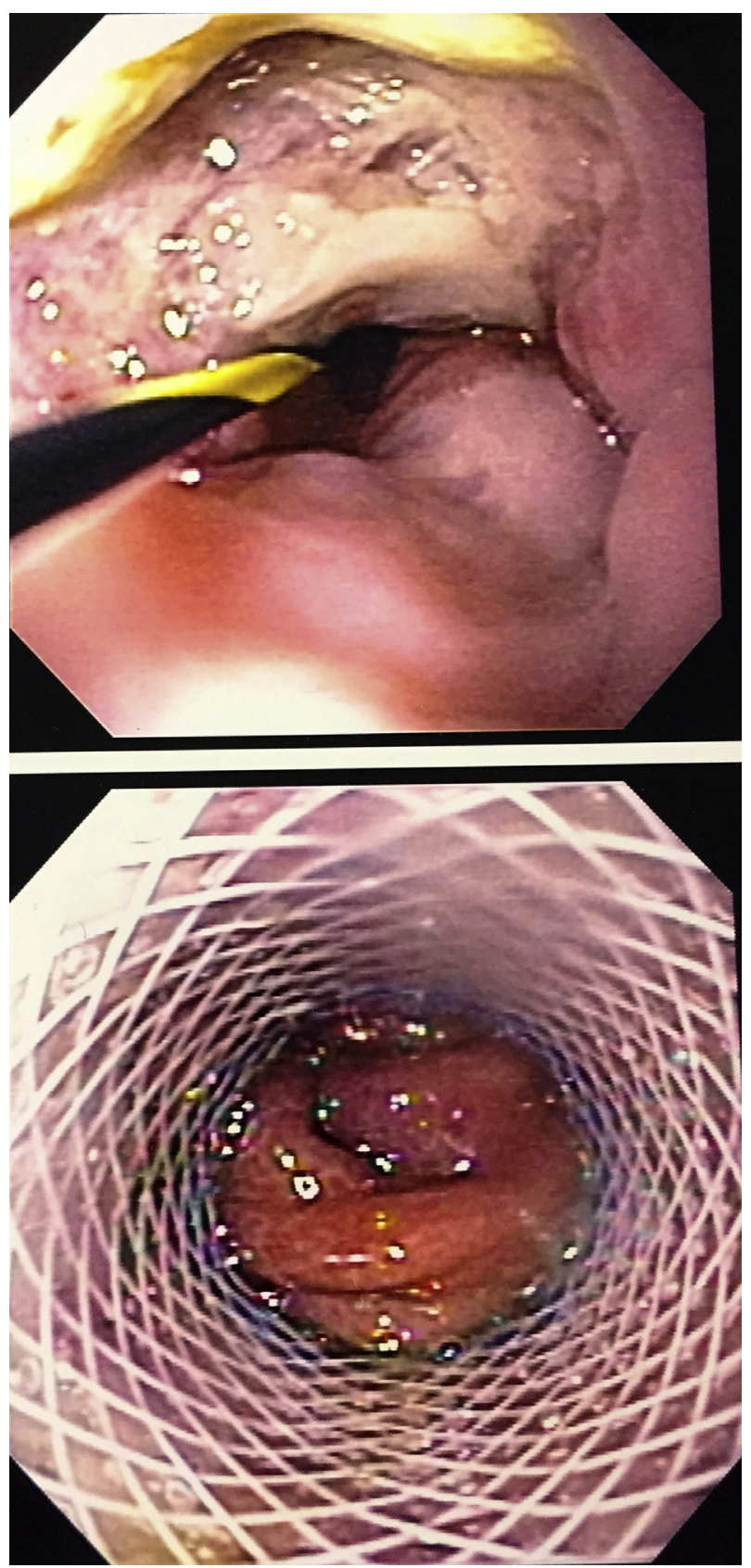

FIGURE 2. An acute esophageal perforation before and after stent placement.

required a similar procedure after their initial surgical repair. Esophageal stents with a Nitinol superstructure were used more frequently than silicone-coated plastic stents.

Table 3 displays the outcomes reviewed for the cohort analysis. Intensive care unit and total length of hospital stay were significantly shorter in the SR group when compared with the OR group. Likewise, the SR group experienced a shorter time interval from stent placement to oral intake than patients undergoing surgical repair. A comparable number of patients in each treatment group were found to have a persistent leak after their initial therapy. However, only 1 patient in the SR group required a subsequent surgical repair, whereas 4 patients in the OR group required reoperative repair. Four patients in the SR group experienced migration of their stent requiring repositioning (1) or replacement (3).

Morbidities included atrial fibrillation (8), wound infection (4), pneumonia (5), myocardial infarction (3), and severe epistaxis (1) in the OR group and pneumonia or a deep venous thrombosis (2) in the SR group. Operative mortalities resulted from a myocardial infarction or sepsis in the OR group and prolonged pneumonia in the SR group (1). After discharge, it was more common for the OR group to require long-term acute care admission, more frequent outpatient encounters, and enteral nutritional support than the SR group.

Readmission was more frequent in the OR treatment group, resulting from pneumonia (2), pleural effusion (2), and atrial fibrillation (1). Two patients in the SR group required readmission for pneumonia or deep vein thrombosis. Dysphagia requiring intervention was also more common in the OR group.

Analysis of mean costs for the 2 treatment groups showed a significant difference (Table 4). Subset analysis revealed that the majority of the cost differences between the 2 cohorts were due to intensive care unit and total length of hospital stay. Mean imaging costs were higher for the SR group than in the OR cohort. Mean costs associated with surgical repair or stent placement were not significantly different.

\section{DISCUSSION}

Operative primary repair of the esophagus, as eloquently described by Whyte and colleagues, ${ }^{8}$ emerged as the preferred method for treating patients with an acute perforation of the intrathoracic esophagus not associated with esophageal necrosis or malignancy since Barrett ${ }^{9}$ reported the first successful repair in 1947. However, despite improvements in surgical technique, anesthesia, critical care, and antimicrobial therapy, the magnitude of transthoracic surgical repair results in significant rates of morbidity, the need for prolonged inpatient, and outpatient phases of treatment and a postoperative leak rate recently reported at $39 \% .{ }^{10} \mathrm{~A}$ persistent leak after OR also may result in the need for esophageal diversion and subsequent reconstruction with its associated significant morbidity and patient dissatisfaction.

Over the last decade, experience with esophageal stent placement for the treatment of esophageal perforation, fistula, and anastomotic leak has been found to be beneficial in selected patients. The use of esophageal stents began to find favor as newer biomaterials allowed the production of stents that were easier to place and remove and were capable of forming an occlusive seal along the esophageal wall. ${ }^{11,12}$ Subsequently, several centers have reported using an esophageal stent for acute perforations of various 
TABLE 1. Comparison of the treatment cohorts after propensity matching

\begin{tabular}{|c|c|c|c|c|}
\hline & $\begin{array}{c}\text { Operative } \\
\text { repair }\end{array}$ & $\begin{array}{l}\text { Operative } \\
\text { repair cohort } \\
\text { before } \\
\text { propensity } \\
\text { matching } \\
\end{array}$ & $\begin{array}{l}\text { Stent } \\
\text { repair }\end{array}$ & $\begin{array}{c}P \\
\text { value } \\
\end{array}$ \\
\hline $\mathrm{N}$ & 30 & 93 & 30 & \\
\hline Age (mean y) & $57 \pm 26$ & $67 \pm 19$ & 61 & .4 \\
\hline \multicolumn{5}{|l|}{ Year of treatment } \\
\hline 2009 & 8 & 23 & 6 & \\
\hline 2010 & 6 & 31 & 8 & \\
\hline 2011 & 9 & 29 & 9 & \\
\hline 2012 & 7 & 10 & 7 & \\
\hline Female & $12(40 \%)$ & $67(72 \%)$ & $11(37 \%)$ & 1 \\
\hline $\begin{array}{l}\text { Charlson Comorbidity } \\
\text { (mean score) }\end{array}$ & $5.6 \pm 0.4$ & $6.1 \pm 1.1$ & $5.9 \pm 0.9$ & .1 \\
\hline \multicolumn{5}{|l|}{ Cause of perforation } \\
\hline Endoscopy with biopsy & 8 & 51 & 7 & \\
\hline Endoscopy/FB removal & 9 & 19 & 10 & \\
\hline Endoscopic antireflux & 2 & 7 & 3 & \\
\hline Dilatation & 9 & 11 & 9 & \\
\hline EMR & 2 & 5 & 1 & \\
\hline $\begin{array}{l}\text { Perforation to treatment } \\
\text { (mean } \mathrm{h} / \text { median) }\end{array}$ & $7 \pm 9 / 6$ & $15 \pm 11 / 13$ & $9 \pm 6 / 8$ & .3 \\
\hline Sepsis at presentation & $6(20 \%)$ & $39(42 \%)$ & $8(27 \%)$ & .76 \\
\hline
\end{tabular}

$F B$, Foreign body; EMR, endoscopic mucosal resection.

types with results that are equivalent or superior to those reported for OR. ${ }^{13-15}$ Although intuitively the results of these series imply the potential for shorter hospital stay, less recuperation, and reduced overall costs when compared with surgical repair, no direct comparison has been reported.

It is not surprising that a randomized trial evaluating esophageal stent placement versus surgical primary repair has not been performed. The incidence of esophageal perforation is low, resulting in an accrual period at any single center that would be excessive. The majority of

TABLE 2. Comparison of factors associated with repair between the treatment cohorts

\begin{tabular}{lcc}
\hline & Operative repair & Stent repair \\
\hline Muscle buttress used & $23(77 \%)$ & - \\
Stent type & - & $19(63 \%)$ \\
$\quad$ Nitinol frame & - & $11(37 \%)$ \\
$\quad$ Silicone coated plastic & - & $24(80 \%)$ \\
Associated procedures & $3(10 \%)$ & $19(63 \%)$ \\
$\quad$ PEG & $19(63 \%)$ & - \\
$\quad$ VATS & $11(37 \%)$ & - \\
Jejunostomy & $3(10 \%)$ & $9(3 \%)$ \\
$\quad$ Gastrostomy & - & $47 \pm 18$ \\
$\quad$ Bronchoscopy & - & \\
Stent dwell time (mean d) & \multicolumn{2}{l}{} \\
\hline$P E G$, Percutaneous endoscopic gastrostomy; VATS, video-assisted thoracoscopic- \\
surgery.
\end{tabular}

TABLE 3. Comparison of outcomes for the 2 treatment cohorts

\begin{tabular}{lccc}
\hline & Operative repair & Stent repair & $\boldsymbol{P}$ value \\
\hline $\mathrm{N}$ & 30 & 30 & \\
ICU stay (mean d) & $4 \pm 3$ & $2 \pm 1$ & .001 \\
Total length of stay (mean d) & $11 \pm 7$ & $6 \pm 3$ & .0007 \\
Oral intake (mean d) & $8 \pm 7$ & $3 \pm 2$ & .0004 \\
Leak after initial repair & $6(20 \%)$ & $5(17 \%)$ & 1 \\
Reoperative repair & $4(13 \%)$ & $1(3 \%)$ & 0.35 \\
Stent migration & - & $4(13 \%)$ & \\
Morbidity & $13(43 \%)$ & $4(17 \%)$ & .02 \\
Mortality & $2(7 \%)$ & $1(3 \%)$ & 1 \\
LTAC admission & $12(40 \%)$ & $4(17 \%)$ & .04 \\
Enteral nutrition after & $18(60 \%)$ & $4(17 \%)$ & .0004 \\
$\quad$ discharge & & & \\
Readmission & $5(17 \%)$ & $2(7 \%)$ & .4 \\
Dysphagia & $8(27 \%)$ & $2(7 \%)$ & .08 \\
\hline ICU, Intensive care unit; $L T A C$, long-term acute care center.
\end{tabular}

$I C U$, Intensive care unit; $L T A C$, long-term acute care center.

such perforations also occur unexpectedly after endoscopy, making an informed consent process for randomization difficult. Stent placement for an esophageal perforation remains an "off label" indication, further complicating the funding of a prospective trial. Last, there would likely be a question of whether the 2 treatment options, as they exist today, have equipoise. Investigators and referring physicians familiar with esophageal stent placement may think that patients randomized to OR could have a worse outcome than patients randomized to the use of a stent.

This investigation sought to compare the outcomes and costs of patients treated by OR or SR after an acute iatrogenic, intrathoracic esophageal perforation. Because of the factors previously outlined regarding the possibility of a randomized controlled trial in this patient population, a multicenter, retrospective study design was used. Propensity matching was used to maximize the homogeneity of the 2 treatment groups. A multicenter approach was chosen in the hope that a significant number of patients treated for an iatrogenic perforation over a relatively brief period of time could be identified.

The propensity-matched comparison of patients with an iatrogenic esophageal perforation treated by $\mathrm{OR}$ or SR found several significant differences in outcomes. Patients undergoing OR experienced a significantly longer intensive care unit and total hospital length of stay

TABLE 4. Cost comparison of operative repair and stent repair patient cohorts

\begin{tabular}{lccc}
\hline & Operative repair & Stent repair & $\boldsymbol{P}$ value \\
\hline $\begin{array}{c}\text { Total costs } \\
\text { (mean dollars) }\end{array}$ & $\$ 142,000 \pm 21,500$ & $\$ 91,000 \pm 11,200$ & $<.0001$ \\
$\begin{array}{c}\text { Total inpatient costs } \\
\text { (mean dollars) }\end{array}$ & $\$ 87,000 \pm 16,300$ & $\$ 59,000 \pm 9200$ & $<.0001$ \\
$\begin{array}{c}\text { Total outpatient costs } \\
\text { (mean dollars) }\end{array}$ & $\$ 55,000 \pm 17,000$ & $\$ 32,000 \pm 8700$ & $<.0001$ \\
\hline
\end{tabular}


when compared with patients undergoing SR. The incidence of persistent leaks after initial repair was similar between the 2 patient cohorts. However, more patients undergoing OR underwent reoperative treatment of their persistent leak.

Patients in the SR cohort were able to initiate oral intake significantly sooner and had a significantly lower rate of requiring enteral feeding at the time of discharge from the hospital when compared with patients in the OR cohort. Patients in the OR cohort also had a significantly higher incidence of requiring admission to a long-term acute care facility at the time of hospital discharge than patients in the SR cohort. Last, patients in the OR group experienced a higher rate of post-repair dysphagia requiring treatment that approached statistical significance.

A comparison of the costs of care associated with the SR and OR cohorts found that regardless of the treatment modality used, iatrogenic esophageal perforation is expensive. Although the majority of these costs were associated with the initial inpatient admission, a significant portion was incurred after discharge. Analyses not including this phase of care will not only underestimate the total costs associated with each treatment but also ignore significant differences in costs between the treatment cohorts.

Subset analysis of costs between the OR and SR groups found that the OR group's costs were higher for both mean intensive care unit and total hospital stay. Patients undergoing SR had higher costs related to imaging than patients undergoing OR. Not predicted were the findings that the OR group had higher costs associated with reoperation, long-term acute care use, readmission, enteral nutrition, and subsequent outpatient treatment for dysphagia.

Although the results of this investigation are compelling, some limitations exist in the comparison of the 2 patient groups. A retrospective analysis was performed, and despite using propensity analysis to generate 2 similar patient cohorts, a prospective, randomized design would have been preferable. However, as previously described, significant obstacles exist to enacting such a protocol.

Additional points of contention include the use of different stents in the SR group and likely individual surgeon and facility idiosyncrasies that influenced the OR cohort. Relatively small absolute numbers of patients were involved in this comparison despite the fact that patients received their treatment at more than a single institution. Although small sample size is a weakness of this review, it is inherent in evaluating the very select subset of patients sustaining an acute, iatrogenic esophageal perforation. Last, the fact that both treatment strategies used for iatrogenic esophageal perforation are likely uncommon procedures at most treatment facilities implies that standardization, even at the local level, may be lacking.

Criticisms of the cost comparison portion of this investigation also exist. Costs were attributed over a 4-year study period. Although one of the matching criteria was calendar year, this time interval may have allowed for inflation of costs despite this investigation into use of mean values for comparison. Patient migration for care outside the Ascension Health system may have occurred, in which case some costs may not have been identified. Although cost data are preferable to charge data as a comparison, parity is being assumed on the basis of the establishment of charge parity between facilities.

\section{CONCLUSIONS}

Esophageal stent placement for the treatment of an acute esophageal perforation seems to be as effective as surgical repair when compared between propensity-matched patients. However, stent placement resulted in a shorter length of stay, lower rates of morbidity, and lower costs when compared with traditional surgical repair. Stent placement also may result in a lower rate of post-repair stenosis and dysphagia when compared with traditional OR.

\section{Conflict of Interest Statement}

Authors have nothing to disclose with regard to commercial support.

You can watch a Webcast of this AATS meeting presentation by going to: http://webcast.aats.org/2014/ files/Wednesday/20140430_826AM_837AM_Richard_Free man.mp4

\section{References}

1. Freeman RK, Van Woerkom JM, Ascioti AJ. Esophageal stent placement for the treatment of iatrogenic intra-thoracic esophageal perforation. Ann Thorac Surg. 2007;83:2003-8.

2. Blackmon SH, Santora R, Schwarz P, Barroso A, Dunkin BJ. Utility of removable esophageal covered self-expanding metal stents for leak and fistula management. Ann Thorac Surg. 2010;89:931-7.

3. Leers JM, Holscher AH. Stenting for esophageal perforation and anastomotic leak. In: Ferguson MK, ed. Difficult Decisions in Thoracic Surgery: An Evidence-Based Approach. London: Springer-Verlag; 2011:279-85.

4. Iezzoni LI. Risk Adjustment for Measuring Health Care Outcomes. Chicago, IL: Health Administration Press; 2003:19-69.

5. Quan H, Sundararajan V, Halfon P, Fong A, Burnand B, Luthi JC, et al. Coding algorithms for defining comorbidities in ICD-9-CM and ICD-10 administrative data. Med Care. 2005;43:1130-9.

6. Pearl J. Causality: Models, Reasoning, and Inference. 2nd ed. New York: Cambridge University Press; 2009:31-76.

7. Parsons L. Reducing Bias in a Propensity Score Matched-Pair Sample Using Greedy Matching Techniques. Proceedings of the 26th Annual SAS Users Group International Conference. Cary, NC: SAS Institute Inc; 2001:214-26.

8. Whyte RI, Iannettoni MD, Orringer MB. Intrathoracic esophageal perforation. The merit of primary repair. J Thorac Cardiovasc Surg. 1995;109:140-6.

9. Barrett NR. Report of a case of spontaneous perforation of the esophagus successfully treated by operation. Br J Surg. 1947;35:216-8.

10. Keeling W, Miller D, Lam G. Low mortality after treatment for esophageal perforation: a single-center experience. Ann Thorac Surg. 2010;90:1669-73.

11. Mumtaz H, Barone GW, Ketel BL, Ozdemir A. Successful management of a nonmalignant esophageal perforation with a coated stent. Ann Thorac Surg. 2002; 74:1233-5.

12. Freeman RK, Ascioti AJ. Esophageal stent placement for perforation, leak or fistula. Semin Thorac Cardiovasc Surg. 2011;23:154-8. 
13. D'Cunha J, Rueth NM, Groth SS, Maddaus MA, Andrade RS. Esophageal stents for anastomotic leaks and perforations. J Thorac Cardiovasc Surg. 2011;42: $39-46$.

14. van Heel NC, Haringsma J, Spaander MC, Bruno MJ, Kuipers EJ. Short-term esophageal stenting in the management of benign perforations. Am J Gastroenterol. 2010;105:1515.

15. Moyes LH, Mackay CK, Forshaw MJ. The use of self-expanding plastic stents in the management of oesophageal leaks and spontaneous oesophageal perforations. Diagn Ther Endosc. 2011;2011:418103.

Key words: esophageal perforation, esophageal stent, cost

\section{Discussion}

Dr N. Christie (Pittsburgh, Pa). Dr Freeman, you and your colleagues should be congratulated on your ongoing efforts to improve our treatment of esophageal perforation using the new technology of esophageal stents.

You had previously identified a subset of patients who were at high risk of failure of treatment with esophageal stent therapy, and that included cervical esophageal perforation, perforation spanning the esophagogastric junction, and an injury greater than $6 \mathrm{~cm}$. You also previously reported the effectiveness of esophageal stenting for spontaneous rupture of the esophagus.

In this comparison study, where did you note a superiority of esophageal stenting with minimally invasive drainage and stenting over thoracotomy? You limited the patients to iatrogenic injury, and the mean time to treatment from the time of injury was approximately 7 to 8 hours. In this subset of patients with presumably minimal mediastinal contamination, one may argue that this is a subset of patients who would be particularly amenable to this stenting therapy. To help guide others in the society who don't have as much experience as you, do you think that these good outcomes with stenting can be generalized to a wider group of patients with esophageal perforation, or what are your selection criteria?

Dr Freeman. The challenge of this study was to try and compare patients with an esophageal perforation, which is difficult, so we thought that the iatrogenic population was more comparable, taking the spontaneous perforations out of that, taking malignancy out of that. So it wasn't an attempt to stack the deck as much as to be able to compare the patients properly. Our initial approach to almost all perforations now is esophageal stent placement. You mentioned the criteria we talked about. We don't try and stent patients who have a perforation greater than 6 $\mathrm{cm}$ in length; otherwise, we look closely at using stent as an initial treatment.
Dr Christie. You reported a relatively low rate of stent migration and need to reintervene. Others have reported on some special techniques to try and prevent stent migration in what are otherwise relatively normal esophagi. From a technical point of view, are there any special techniques that you have used to try and prevent migration?

Dr Freeman. We tend to use the same technique that we have used from the beginning, which is to oversize the stent in both length and diameter. We think this helps and are leaving the stent in for shorter and shorter periods of time.

Dr Christie. Thank you.

Dr B. Meyers (St Louis, Mo). This use of propensity matching has a great potential to move the analysis along to the next level. Many of your other presentations have been single-center case series. I wonder, though, if the propensity matching doesn't lack some variables that might be telling or influential. I imagine that you are the primary provider of the stents in this study, because it is your hospital system, and there is likely to be a strong "surgeon effect." Your personal expertise in this care might not translate into the conclusion that other people use the stent as effectively as a tool. That is 1 question, which is a compliment and a question at the same time.

Do the matching variables account for the fact that there might be subjectivity at the low end of the range of severity of perforation, that is, in patients in whom stents might be used by some practitioners in situations that might actually not be treated with a thoracotomy and repair? The alternative to stent in some cases might be observation or treatment with some other milder intervention? Even with the propensity matching, you might be comparing apples to oranges if very mild stented cases are included.

Dr Freeman. The stent cases came from 3 different hospitals. We tried to limit the number of these patients who came from our institution for exactly the reason that you stated. So they are not all from one institution on the stent side. There are probably variables that we will never know about at the granular level as far as who receives stents and who receives surgery and how that decision is made. What I would say is that it was interesting as we went over all the patients who were in this study. I think if we would have done this study 10 years ago, we would have found patients who had perforations that maybe were contained and small, and the patient had been nil per os. We didn't see any of those patients. I wonder if one of the side effects of talking about stenting is that those patients are now treated just with observation and antibiotics. All of these patients had clear perforations with flow into the pleura or mediastinum. 